\title{
Universal Pareto Dominance and Welfare for Plausible Utility Functions
}

HARIS AZIZ, NICTA and University of New South Wales, Sydney, Australia and FLORIAN BRANDL, Technische Universität München, 85748 Garching, Germany and FELIX BRANDT, Technische Universität München, 85748 Garching, Germany

Categories and Subject Descriptors: F.2.2 [Analysis of Algorithms and Problem Complexity]: Nonnumerical Algorithms and Problems-Computations on discrete structures; J.4 [Computer Applications]: Social and Behavioral Sciences-Economics

Additional Key Words and Phrases: SSB utility, stochastic dominance, efficiency welfare theorem

Perhaps one of the most fundamental notions in economics is that of Pareto efficiency. We study Pareto efficiency in a setting that involves two kinds of uncertainty: Uncertainty over the possible outcomes is modeled using probability distributions (lotteries) whereas uncertainty over the agents' preferences over lotteries is modeled using sets of plausible preference relations. A lottery is universally undominated if there is no other lottery that Pareto dominates it for all plausible preference relations. A lottery is potentially efficient if it is Pareto efficient for some vector of plausible preference relations. It is easily seen that every potentially efficient lottery is universally undominated.

We show that, under fairly general conditions, the converse holds as well, i.e., the set of universally undominated and the set of potentially efficient lotteries coincide. Special cases of our result include the ordinal efficiency welfare theorem by McLennan [2002] and a generalization of the latter by Carroll [2010]. In contrast to their work which is based on von Neumann-Morgenstern (vNM) utility theory, we assume that preferences over lotteries are given by sets of skew-symmetric bilinear (SSB) utility functions. SSB utility theory is a generalization of $\mathrm{vNM}$ utility theory that neither requires transitivity nor the controversial independence axiom [see, e.g., Fishburn 1988].

Sets of plausible utility functions are typically interpreted as incomplete information on behalf of the social planner. Indeed, it seems quite natural to assume that the social planner's information about the agents' utility functions is restricted to ordinal preferences, top choices, or subsets of pairwise comparisons. Further conditions might be implied by domain restrictions. Three particularly interesting classes of plausible utility functions arise when contemplating that only ordinal preferences over pure outcomes are known. For a given preference relation $R_{i}$, we consider

- the set of all SSB functions consistent with $R_{i}$,

- the set of all vNM functions consistent with $R_{i}$, and

- the (unique) canonical SSB function consistent with $R_{i}$.

Author's addresses: Haris Aziz, NICTA and UNSW, Kensington 2033, Australia, Email: haris.aziz@nicta.com.au, Florian Brandl and Felix Brandt, Technische Universität München, 85748 Garching, Germany, Email: \{brandlf, brandtf \}@in.tum.de

Permission to make digital or hard copies of part or all of this work for personal or classroom use is granted without fee provided that copies are not made or distributed for profit or commercial advantage, and that copies bear this notice and the full citation on the first page. Copyrights for third-party components of this work must be honored. For all other uses, contact the owner/author(s). Copyright is held by the author/owner(s).

EC'14, June 8-12, 2014, Stanford, CA, USA.

ACM 978-1-4503-2565-3/14/06.

http://dx.doi.org/10.1145/http://dx.doi.org/10.1145/2600057.2602866 
These sets give rise to three natural extensions of preferences over alternatives to preferences over lotteries and thereby to three notions of ordinal efficiency. While the second notion is equivalent to the well-studied notion of stochastic dominance ( $S D$ efficiency), the other two notions, one weaker and one stronger than $S D$-efficiency, have not been studied before. We call the weaker notion bilinear dominance (BD-efficiency) and the stronger one pairwise comparison ( $P C$-efficiency). The $P C$ preference relation seems particularly natural because it prescribes that lottery $p$ is preferred to lottery $q$ iff it is more likely that $p$ yields a better alternative than $q$. In contrast to the other preference extensions, the $P C$ extension always yields a complete preference relation. However, $P C$ preferences cannot be modeled by any set of linear vNM utility functions.

Additionally, we investigate geometric as well as computational properties of efficiency notions obtained via universal domination. Our findings include the following observations (see also Table I).

- Whether a lottery is $B D$-efficient or $S D$-efficient only depends on its support.

- The set of $S D$-efficient lotteries and the set of $P C$-efficient lotteries may fail to be convex. As a consequence, for both of these notions, the convex combination of two efficient mechanisms may violate efficiency.

- It can be verified in polynomial time whether a given lottery is universally undominated for all notions of ordinal efficiency mentioned in the paper.

- An $S D$-efficient lottery that $S D$-dominates a given lottery can be found in polynomial time.

- For $B D$-efficiency, all considered computational problems can be solved in linear time due to a combinatorial characterization of $B D$-efficiency in terms of undominated sets of vertices in the corresponding Pareto digraph.

- It is possible that there is no $P C$-efficient lottery that $P C$-dominates a given lottery due to cyclical dominations.

\begin{tabular}{l} 
Table I. Properties of varying notions of ordinal efficiency. An efficiency notion sat- \\
isfies convexity if the convex combination of two efficient lotteries is efficient. An \\
efficiency notion is combinatorial if a lottery is efficient iff every lottery with the same \\
support is efficient. An efficiency notion allows efficient improvements if for any given \\
lottery, there is an efficient lottery that dominates the original lottery. \\
\hline \begin{tabular}{cccc}
\multicolumn{2}{l}{ convex } & combinatorial & allows efficient improvements \\
\hline BD-efficiency & + & + & + \\
ex post efficiency & + & + & + \\
$S D$-efficiency & - & + & + \\
$P C$-efficiency & - & - & + \\
\hline
\end{tabular}
\end{tabular}

\section{Acknowledgments}

This material is based upon work supported by Deutsche Forschungsgemeinschaft under grants BR 2312/7-2 and BR 2312/10-1 and by NICTA which is funded by the Australian Government through the Department of Communications and the Australian Research Council through the ICT Centre of Excellence Program.

\section{REFERENCES}

CARroll, G. 2010. An efficiency theorem for incompletely known preferences. Journal of Economic Theory 145, 6, 2463-2470.

FishBURN, P. C. 1988. Nonlinear preference and utility theory. The Johns Hopkins University Press.

MCLENNAN, A. 2002. Ordinal efficiency and the polyhedral separating hyperplane theorem. Journal of Economic Theory 105, 2, 435-449. 